\title{
Origin of Atomic Clusters during Ion Sputtering
}

\author{
L. E. Rehn, R. C. Birtcher, S. E. Donnelly, P. M. Baldo, and L. Funk \\ Materials Science Division, Argonne National Laboratory, Argonne, Illinois 60439
}

(Received 19 June 2001; published 24 October 2001)

\begin{abstract}
Previous studies have shown that the size distributions of small clusters $(n \leq 40 ; n=$ number of atoms/cluster) generated by sputtering obey an inverse power law with an exponent between -8 and -4 . Here we report electron microscopy studies of the size distributions of larger clusters $(n \geq 500)$ sputtered by high-energy ion impacts. These new measurements also yield an inverse power law, but one with an exponent of -2 and one independent of sputtering yield, indicating that the large clusters are produced when shock waves, generated by subsurface displacement cascades, ablate the surface.
\end{abstract}

DOI: $10.1103 /$ PhysRevLett.87.207601

The emission of clusters of atoms during ion sputtering was first reported more than 40 years ago [1]. This observation of stable, intact clusters elicited considerable surprise because the energies involved in the ion-target collisions that generate sputtered atoms are typically much larger, ranging up to many $\mathrm{keV}$, than the $1-2 \mathrm{eV}$ binding energies holding the clusters together. Hence it appears difficult to understand how stable clusters can form during ion sputtering. Ever since their discovery, the unsolved fundamental question of the cluster emission mechanism has attracted considerable experimental and theoretical attention; recent reviews can be found in the literature [2-4].

Mass spectrometry has been used extensively in prior experimental studies of cluster emission during sputtering primarily because this technique allows the size distributions of clusters, at least for those comprising up to about 40 atoms, to be determined both accurately and conveniently [2-6]. Since there were serious concerns regarding possible fragmentation during measurement, particularly for larger clusters, and since larger clusters cannot be monitored as efficiently with mass spectrometry, it appears that only populations of smaller clusters have been investigated previously. It has been shown $[5,6]$ that the size distributions, $Y(n)$, of the emitted clusters obey an inverse power law, i.e.,

$$
Y(n) \propto n^{-\delta},
$$

where $n$ is the number of atoms in a given cluster. The total sputtering yield is defined as the total number of atoms emitted from the surface per incoming ion, regardless of whether they are emitted individually or in clusters. The exponent, $\delta$, was found to correlate [4-6] with the total sputtering yield, such that higher sputtering yields result in smaller values of $\delta$. The values of $\delta$ that have been determined experimentally using mass-spectrometry techniques, that is, for those clusters containing up to approximately 40 atoms, range between 4 and $8[5,6]$.

No simple theoretical model has been able to reproduce both the power-law size distributions and the high values of $\delta$ found experimentally. Statistical models, which assume that the observed intact clusters arise from the reaggregation of atoms after they have been ejected individually from
PACS numbers: 79.20.Rf, 61.80.Jh

the surface, and which would appear most appropriate for predicting the distribution of the smaller clusters, yield an exponentially decaying number of clusters with increasing cluster size $[2,4]$. A power-law distribution is predicted by the shock-wave model of Bitensky and Parilis [7], and an approximate one by the thermodynamic-equilibrium model of Urbassek [8]. However, these models yield values of 2 and $7 / 3$, respectively, for the $\delta$ in Eq. (1), and thus they have been rejected based upon the previously reported experimental results lying between 4 and 8 .

In this Letter, we report transmission electron microscopy (TEM) studies of the size distributions of the larger clusters $(n \geq 500)$ that are sputtered from the surface by high-energy ion impacts. Consistent with the previous reports, these new measurements also yield an inverse power law for the cluster size distribution. However, these new measurements yield an exponent of -2 in the power law, and one that is independent of total sputtering yield. As discussed in more detail below, this inverse-square dependence reveals that the clusters are produced when shock waves, generated by subsurface displacement cascades, impact and ablate the surface, as originally proposed in the model of Bitensky and Parilis [7]. Many smaller clusters can result from the breakup of these larger ones, which provides a plausible explanation for the large negative exponents that have been reported previously using massspectrometry methods.

The experiments were performed as follows. Au films, $50 \pm 5 \mathrm{~nm}$ thick with a $\langle 110\rangle$ surface normal, were deposited by $e$-beam evaporation of 99.999 at. \% Au onto $\mathrm{NaCl}$ substrates held at a temperature of $350{ }^{\circ} \mathrm{C}$. Pieces of the evaporated $\mathrm{Au}$ films were floated off the $\mathrm{NaCl}$ in a water/alcohol mixture and sifted onto $\mathrm{Cu}$ TEM grids. The ion sputtering experiments were performed in the Hitachi $\mathrm{H}-9000$ electron microscope operating at $300 \mathrm{kV}$, located in the IVEM-Tandem facility at Argonne National Laboratory [9]. The ion beam was oriented $30^{\circ}$ from the microscope axis, and the specimen was tilted $15^{\circ}$ toward the ion beam so that both ions and electrons were incident on the specimens at $15^{\circ}$ to the foil normal. The ions passed first through the 50-nm thick gold foil target pieces, then through a TEM transparent carbon-coated collector grid 
that was positioned approximately $30 \mu \mathrm{m}$ below the target foil. Hence this is a transmission sputtering experiment, in contrast to the reflection geometry commonly employed in mass-spectrometry studies. The transmission geometry was chosen because it provides superior in situ images of the collected particles. Although the size distributions were not analyzed in detail, we did observe similar sized particles in the reflection geometry.

Sputtering was performed with a series of ions $(\mathrm{Ne}, \mathrm{Ar}$, $\mathrm{Kr}$, and $\mathrm{Au}$ ) of increasing atomic number at energies between 400 and $500 \mathrm{keV}$, i.e., at energies higher than those typically used in mass-spectrometry studies. The target and collector foils were at room temperature, and the ion flux was maintained between $(1-2) \times 10^{15}$ ions $/ \mathrm{m}^{2} / \mathrm{s}$. The beam current was monitored continuously during the sputtering experiments using an annular Faraday cage that was calibrated via a removable Faraday cup having an absolute accuracy estimated to be $\pm 10 \%$. The total ion fluence was kept low, less than $1 \mathrm{ion} / \mathrm{nm}^{2}$, in order to minimize any potential complications from either surface roughening, target thinning, or resputtering of the collected particles.

A bright-field TEM micrograph of the particles collected on the carbon-coated grid following a $400-\mathrm{keV} \mathrm{Kr}$ irradiation to a fluence of $1 \times 10^{18}$ ions $/ \mathrm{m}^{2}$ is shown in Fig. 1. The sizes of the collected particles are broadly distributed, from just barely discernible images somewhat less than a $\mathrm{nm}$ in lateral dimension up to particles as large as approximately $8 \mathrm{~nm}$ in extent. The TEM imaging conditions were adjusted so that the contrast shown in Fig. 1 arises primarily from differences in mass. Hence the fact that the larger particles are noticeably darker in the micrograph demonstrates that the particles are three-dimensional entities. The three-dimensional nature of the collected particles was subsequently confirmed

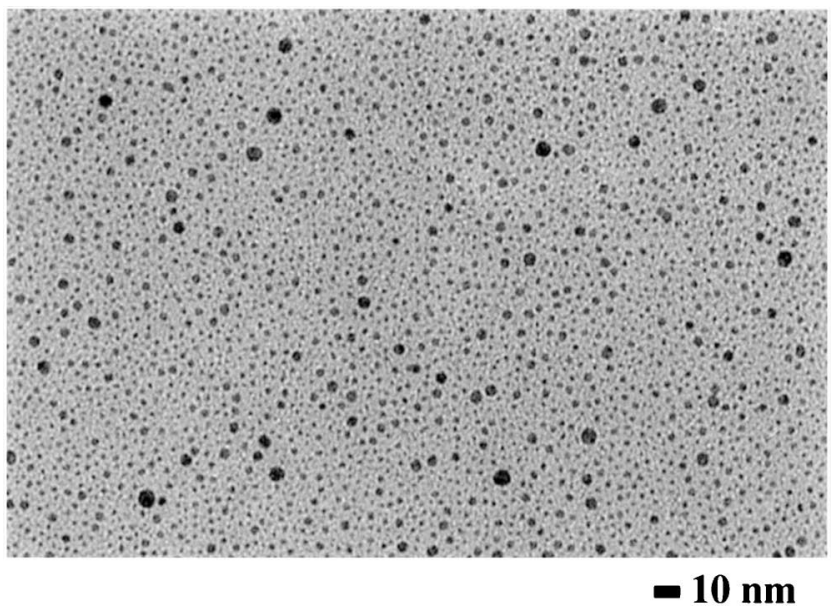

FIG. 1. Bright-field TEM micrograph of Au particles collected on a carbon-coated grid following a $400-\mathrm{keV} \mathrm{Kr}$ irradiation to a fluence of $1 \times 10^{18}$ ions $/ \mathrm{m}^{2}$. Note that the larger particles are noticeably darker, demonstrating that the particles are threedimensional entities. from high-resolution TEM images taken in a JEOL4000 microscope.

The particle size distribution, $Y(n)$, i.e., the number of clusters containing $n$ atoms, was determined after removing the Au target foil as follows. First, an image similar to the one shown in Fig. 1 was digitized using a Leafscan 45 at a final image resolution of 10 pixels $/ \mathrm{nm}$. Sizes of individual nanoparticles were obtained from the scanned images using the computer program Photoshop. The measured lateral dimensions were converted into the number of atoms for a given cluster by assuming that the particles were hemispheres with the density of bulk Au. All visible particles in an area of $\sim 10^{5} \mathrm{~nm}^{2}$ were sized, i.e., approximately 650 and 200 particles, respectively, for the Au and the Ne irradiation. The results were grouped into bins having 0.5-nm steps; the procedure was repeated following each irradiation. As reported previously [10], in situ video recordings showed that the sputtered clusters arrived at the collector foil well separated, both temporally and spatially. The total nanoparticle density determined by counting individual nanoparticles in the scanned images had a one-to-one correlation with the integrated arrival rates determined from the in situ video recordings. Subsequent irradiation of the particles without a target foil did not produce particle growth. Taken together these findings demonstrate that size changes following deposition were insignificant.

The size distributions that were obtained in this manner are displayed in Fig. 2. Size distributions were determined for the particles collected from the 400-keV Kr irradiation shown in Fig. 1, and for 400-keV Ne and Au irradiations to

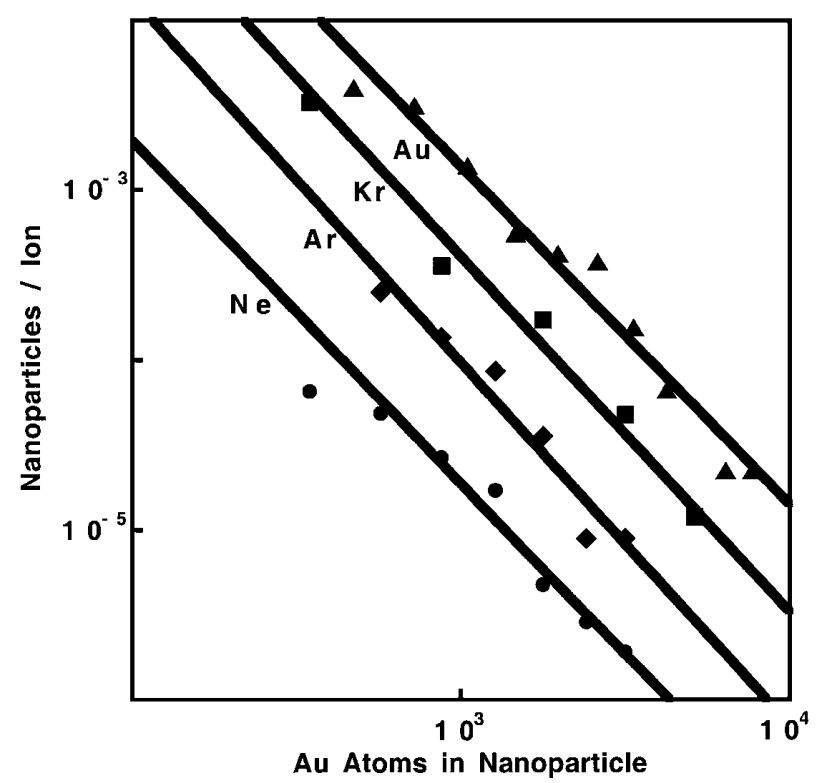

FIG. 2. Measured number of collected clusters as a function of cluster size $(n)$ for the $400-\mathrm{keV} \mathrm{Kr}$ irradiation shown in Fig. 1, and for $400-\mathrm{keV} \mathrm{Ne}$ and $\mathrm{Au}$ irradiations to fluences of $6.2 \times$ $10^{18}$ ions $/ \mathrm{m}^{2}$ and $3.8 \times 10^{17}$ ions $/ \mathrm{m}^{2}$, respectively, and for a $500-\mathrm{keV}$ Ar irradiation to a fluence of $2 \times 10^{18} \mathrm{ions} / \mathrm{m}^{2}$. 
fluences of $6.2 \times 10^{18}$ ions $/ \mathrm{m}^{2}$ and $3.8 \times 10^{17}$ ions $/ \mathrm{m}^{2}$, respectively, and for a $500-\mathrm{keV}$ Ar irradiation to a fluence of $2 \times 10^{18}$ ions $/ \mathrm{m}^{2}$. Two important findings emerge from this compilation. First, the displayed power-law fits to the data from the four individual irradiations all have the same slope despite large differences in the total sputtering yield (e.g., $\sim 5$ for the $\mathrm{Ne}$ and $>100$ for the $\mathrm{Au}$ ). Second, the magnitude of this slope is -2 within remarkably tight error limits; the average standard deviation determined for the four runs was $<0.05$.

Early after their discovery, it was recognized that a mechanism involving the cooperative motion of atoms was probably responsible for the emission of intact clusters. Expanding on this concept, and noting that clusters were formed with the greatest probability under energetic heavy-ion bombardment, Bitensky and Parilis [7] formulated an analytical model for cluster emission based upon the formation and emergence of shock waves that originated from energetic subsurface collision cascades. Essentially, the arrival and reflection of a shock wave at a sample surface places the latter under tension. If this tension exceeds a critical value, known as the fracture strength, the surface fractures and fragments are ejected. While ejection of near-surface material has been observed from the shock wave created along the path of an ion traversing just beneath a solid surface [11], the shock wave invoked in the Bitensky and Parilis model is a different one, one created by the rapid increase in temperature and pressure that occurs following an individual energetic displacement event [12]. The Bitensky and Parilis model predictions are in excellent agreement with the present results (Fig. 2). Their shock-wave model predicts a powerlaw decrease for the cluster size distribution, and one with an exponent of -2 . This inverse-square dependence arises because the critical parameter in the fracture process is the energy required to create new surface area, which for a three-dimensional particle increases as its characteristic dimension squared.

Although the thermodynamic-equilibrium model of Urbassek [8] yields approximately a power-law decrease, its exponent of $7 / 3$ lies outside the experimental results depicted in Fig. 2. Some "flattening" of the particles upon impact would tend to increase the measured exponent; however, this appears insufficient to reach the $7 / 3$ prediction. Moreover, in order for large clusters to be emitted this model requires that the near-surface irradiated volume pass through the liquid-gas coexistence regime at the point when the thermalized cascade core expands into the vacuum. These assumptions [8] of "criticality," following thermalization, cannot be expected to remain valid over the wide range of ion masses (total sputtering yield), and large particle sizes, for which the inverse-square size dependence has been observed in the present set of experiments. For these reasons, the emission of the large clusters is attributed to the shock-wave mechanism of Bitensky and Parilis [7].
We turn next to the results obtained previously from mass-spectrometry measurements of smaller particles, namely, the reported exponents ranging between approximately -4 and -8 . As an aid to the discussion, Fig. 3 shows a plot of such exponents reported recently by Staudt, Heinrich, and Wucher [13] for sputtering of a clean Ag surface. The results in Fig. 3 have been plotted as a function of the total sputtering yield (taken from [14]). The Ag data (filled circles) shown in Fig. 3 represent the most convincing evidence obtained to date that the power-law exponent for smaller clusters exhibits a systematic dependence on the total sputtering yield, as first suggested by Coon et al. [5] and Wucher et al. [6]. Our new data are represented by the open circles in Fig. 3; the datum from the Au irradiation at $(105,-2.02)$ has been omitted in order to better illustrate the functional dependence of $\delta$ on total sputtering yield revealed by the Ag data.

The two different data sets have been labeled $n<40$ (solid circles) and $n>500$ (open circles) to emphasize the fact that these exponents were obtained by observing two different regions of the sputtered cluster-size distribution. Plotting the data in this manner makes it straightforward to see the relationship between the two different sets of exponents. The TEM data (open circles; $n>500$ ) represent the size distributions generated by the most energetic ion-target collision events and clearly follow the shock-wave predictions; i.e., they consistently yield an exponent of -2 . It can be seen from Fig. 3 that a simple extrapolation of the Ag data (filled circles; $n<40$ ) to higher sputtering yields also appears to attain the characteristic inverse-square dependence predicted by the shock-wave model. That is, as the sputtering events on average become more energetic, as indicated by the increased sputter

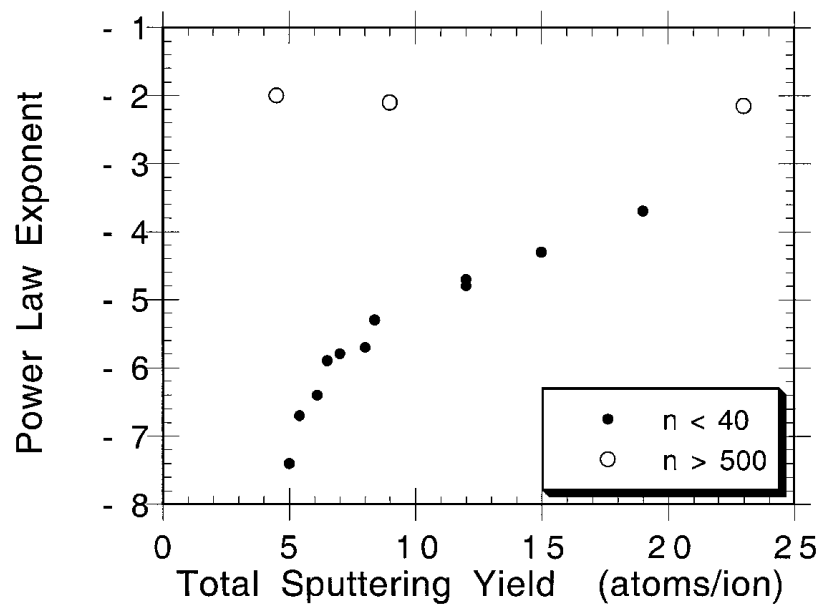

FIG. 3. Plot of the power-law exponents describing the size distribution of sputtered Ag clusters containing less than 40 atoms/cluster obtained using mass spectrometry by Staudt et al. [13], along with those obtained in the present experiment for Au clusters containing $>500$ atoms per cluster, both plotted as a function of total sputtering yield (taken from [14]). 
yield, more of the subsurface displacement cascades generate shock waves strong enough to exceed the surface fracture strength. Hence with increasing sputter yield, the Ag data appear to approach the inverse-square dependence characteristic of shock-wave production. Both data sets therefore provide compelling evidence that the larger clusters are generated by shock waves emitted from the most energetic displacement cascades.

The experimental verification that relatively large atom clusters, ones containing from roughly 500 to 10000 atoms, are generated during high-energy ion bombardment also offers a possible explanation for why the previous reported investigations yielded power-law size distributions with considerably higher exponents. Very simply, a higher exponent means that smaller clusters are present with disproportionately higher probabilities in the mass spectrometry studies. Such an excess of smaller clusters is of course what would occur if all the larger clusters do not remain completely intact. As alluded to in the introduction, the very fact that the interatomic bond energy is so small in comparison to the energies involved in the displacement events implies that some clusters can be expected to fragment after being ablated. In fact, the literature $[2,4-6,13]$ on cluster emission is replete with discussions of cluster instabilities, including as well the difficulties in successfully ionizing a cluster without inducing its fragmentation. When clusters fragment, the distortion in the size distribution will be amplified at the smallest sizes simply because one large cluster can break up into many smaller ones. Note that this statement is not meant to imply that the actual size distributions measured for the smaller clusters can be understood solely in terms of the fragmentation of the larger clusters. Other processes, e.g., direct emission, reaggregation, and the thermodynamic-equilibrium ejection process discussed by Urbassek [8], can also contribute to the population of smaller clusters. Nevertheless, the fact that the measured power-law exponents are systematically higher for smaller clusters is to be expected when particle fragmentation occurs.

In summary, TEM measurements of the larger clusters $(n \geq 500)$ that are sputtered from the surface by highenergy ion impacts reveal an inverse power-law size distribution with an exponent of -2 that is independent of the total sputtering yield. This inverse-square dependence indicates that the larger clusters are formed when shock waves, generated by subsurface displacement cascades, impact and ablate the surface, as predicted in the previously published model of Bitensky and Parilis [7]. Many smaller clusters can result from the putative fragmentation of larger ones, which may explain the much larger negative exponents that have been reported previously. An important corollary from these results is that the nanoparticles which are generated consist of simple fragments of the original surface, that is, ones that have not undergone any large thermal excursions.

We thank Dr. R. Csencsits for assistance with the highresolution electron microscopy work. This research was supported by the U.S. Department of Energy, Office of Science, BES-Materials Science, under Contract No. W-31109-Eng-38.

[1] R. E. Honig, J. Appl. Phys. 29, 549 (1958).

[2] W. O. Hofer, in Sputtering by Particle Bombardment III, edited by R. Behrisch and K. Wittmaack (Springer, Berlin, 1991), p. 15.

[3] K. Nordlund, J. Keinonen, M. Ghaly, and R. S. Averback, Nucl. Instrum. Methods Phys. Res., Sect. B 148, 74 (1999).

[4] A. Wucher and M. Wahl, Nucl. Instrum. Methods Phys. Res., Sect. B 115, 581 (1996).

[5] S. R. Coon, W. F. Calaway, J. W. Burnett, M. J. Pellin, D. M. Gruen, D. R. Spiegel, and J. M. White, Surf. Sci. 259, 275 (1991).

[6] A. Wucher, M. Wahl, and H. Oechsner, Nucl. Instrum. Methods Phys. Res., Sect. B 82, 337 (1993).

[7] I. S. Bitensky and E. S. Parilis, Nucl. Instrum. Methods Phys. Res., Sect. B 21, 26 (1987).

[8] H. M. Urbassek, Nucl. Instrum. Methods Phys. Res., Sect. B 31, 541 (1988).

[9] C. W. Allen, L. L. Funk, E. A. Ryan, and S. T. Ockers, Nucl. Instrum. Methods Phys. Res., Sect. B 40/41, 553 (1989).

[10] R. C. Birtcher, S. E. Donnelly, and S. Schlutig, Phys. Rev. Lett. 85, 4968 (2000).

[11] J. Ronchi, J. Appl. Phys. 44, 3575 (1973).

[12] K. Nordlund, J. Keinonen, M. Ghaly, and R. S. Averback, Nature (London) 398, 49 (1999).

[13] C. Staudt, R. Heinrich, and A. Wucher, Nucl. Instrum. Methods Phys. Res., Sect. B 164/165, 677 (2000).

[14] H. H. Andersen and H. L. Bay, in Sputtering by Particle Bombardment I, edited by R. Behrisch (Springer, Berlin, 1981), p. 145. 